\title{
CHALLENGES IN THE AUTOMOTIVE JIS/JIT PRODUCTION OF STEERING WHEELS INVOLVING TRACEABILITY
}

\author{
Horea Mizgan $^{1}$, Ovidiu Ambruş ${ }^{2}$ \\ University of Oradea \\ 1 Universității Street, 410087 Oradea, Romania \\ ${ }^{1}$ hm@inteliform.ro \\ 2ovidiu.ambrus@litens.com
}

\begin{abstract}
The purpose of this article is to present the new challenges faced by the automotive industry and specifically by the steering wheel producers in terms of product design changes, process changes and the solution to solve them, the concept of TTM - Total Traceability Management. The article presents the traceability solution as a proved solution for managing JIS (Just in Sequency) \& JIT (Just in Time) manufacturing concepts in terms of customer demand sequence attainment, process sequence attainment, and technical test verifications. The research is based on case studies deployed in production facilities, in the companies TRW and Key Safety Systems. Both entities are steering wheel producers with JIS\&JIT conditioned production systems and are also using latest technology in synchronizing the traceability communication with various cars producers (Porsche, BMW, Daimler, etc.). This concept of manufacturing and delivery of the products to the famous automotive companies is crucial for the supplier including the base of business, because the entire work in this domain is completely automatic by robots. That means: all the final products (cars end others), including the components from different suppliers, also the technology of assembling, it need to be adapted in order to be performed by robots. The components as steering wheels, and many others, arrive at the assembling line at OEMs (Original Equipment Manufacturing) facilities and the robots work on directly the buffers with these components in order to ensure the continuous assembling process in the condition of total flexibility and without errors.
\end{abstract}

Keywords: TM-Total Traceability Management, IoT-Internet of Things, JIS-Just in Sequence, JIT-Just in Time

\section{Introduction}

The steering wheel production is passing through significant changes, following the general trend of the automotive industry. The Just in Time (JIT), Just in Sequence (JIS) [1,2], Industry 4.0 [3-6] and Internet of Things [7] are rapidly overtaking also the steering wheel commodity. If in 1920 the steering wheel was formed of a steel armature and a wooden shell (Fig. 1), in 2020 the market demands a much higher complexity steering wheel with the following composition (as shown in the same figure 1). Here we are talking about (C) 2021 Published by University Press. This is an open access article under the CC BY-NC-ND license (http://creativecommons.org/licenses/CC-BY-4.0/) of the lot of types of steering wheels (about over 1600 different type pro year), at many clients (over 30), with variable manufacturing every days. JIT means "Just in Time" (all the containers arrive with few days before the requested date of assembling, and JIS means "Just in Sequence" (the content of which container need to corresponds synchronously with the successive order of the robotized line of the OEM [8]. The base technology is composed of [9]:

- Die casted armature that is built to offer resistance and rigidity to the steering wheel; 
- Injected foam that is the design to give the softness and leather adhesion;

- Sewed leather, that is the foam protector and establishes the product aspect;

- Assembled heating element, used for winter season to add heating comfort;

- Assembled HOD sensor, safety sensor with alarm when hands are off the wheel;

- Assembled chromed or painted bezel, designed as aspect element;

- Assembled multifunctional switches, designed for communication with the car computer;

- Assembled vibration engine, acting as alarm through vibration;

- Assembled electronic control unit, design to control the steering wheel components in relationship with the rest of the car $[10,11]$.

The technology corresponds with variable conditions.

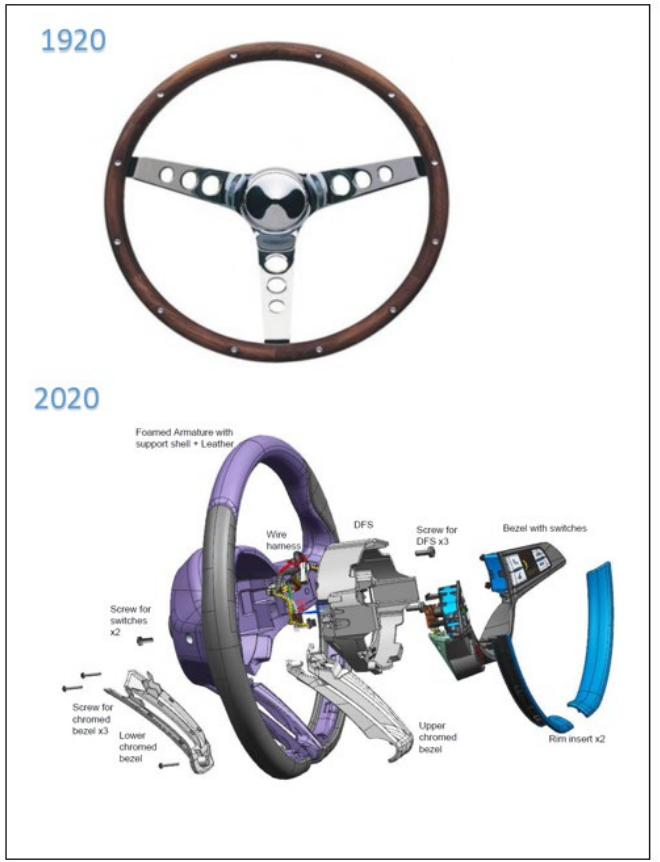

Fig.1: Steering wheel comparison 1920-2020

\section{Process analysis and parameters traced}

As per AIAG FMEA (Automotive Industry Action group - Failure Mode and Effects Analysis) Manual, there are two different types of special characteristics [12]:

- Critical Characteristics (CC), are characteristics that may affect the:

- Safety of product or operator;

- Compliance with legal requirement;

- Environmental requirements.

These characteristics are designated in the D - or PFMEA, and can have a causal relationship to the effect of potential failure modes rated 9-10 for severity.

The manufacturing process (producer and/or suppliers) may have an influence on characteristics which can result in a $\mathrm{CC}$ and may require special control to maintain the required process capability and customer requirements.

- Significant Characteristics (SC) are characteristics that may affect:

○ form, fit or function of the product;

$\circ \quad$ the assembly process (final product/set);

o the production process

These characteristics are designated in the D - or P - FMEA and can have a causal relationship to the effect of potential failure modes rated 5-8 for severity, or where agreed by the cross-functional team, having severity rated less than 5 .

The manufacturing process (producer and/or suppliers) may have an influence on characteristics which can result in an SC and may require special control to maintain the required process capability and customer requirements

The characteristics $\mathrm{CC} / \mathrm{SC}$ are monitored through quality/functionality tests and verifications that must be done as a quality automated request for a steering wheel in various steps of production $[13,14]$ :

1. magnesium alloy composition;

2. die casting process control parameters;

3. porosity and cracks inspection results for the armature;

4. dimensional measurement;

5. nut pressing force;

6. nut presence;

7. foaming injection process control parameters;

8. foaming chemical analysis;

9. foaming hardness;

10. dimensional measurement foaming steering wheel;

11. leather elongation;

12. glue to leather adhesion;

13. glue to foam adhesion;

14. screwing torque;

15. screwing angle;

16. function on off for every switch;

17. pressing force for every switch;

18. illumination for every switch;

19. rotary switch function for every position;

20. hands off detection;

21. heating element resistance;

22. part number verification;

23. allocation of the steering wheel in the customer mobile storage locations.

The characteristics are gathered through change of statuses in the TTM databases as per figure 2: status allocation. For each process step completed we have a change of status in the traceability database:

- Foaming - status 10

- Foaming Preparation - status 15

- Leather wrapping - status 15

- $\quad$ Finishing - status 21

- Subassembly - status 22-29

- General Inspection - status 40

- Packing - status 50. 


\section{JIS\&JIT in Traceability}

The challenges with the new products are:

- Customer wants JIS (Just-In-Sequence) \& JIT (Just-In-Time) production process and delivery;

- The number of production processes increased;

- The number of functions of the product increased;

- The steering wheel incorporates electronics that must communicate with the car;

- Strong increase in quality requirements regarding functionality and visual aspects;

- The number of components in the steering wheel grew.

The JIS/JIT Traceability module is working based on the next operations flow [15]:

- Customer send JIS Sequence Request;

- $\quad$ Producer checks the sequence received;

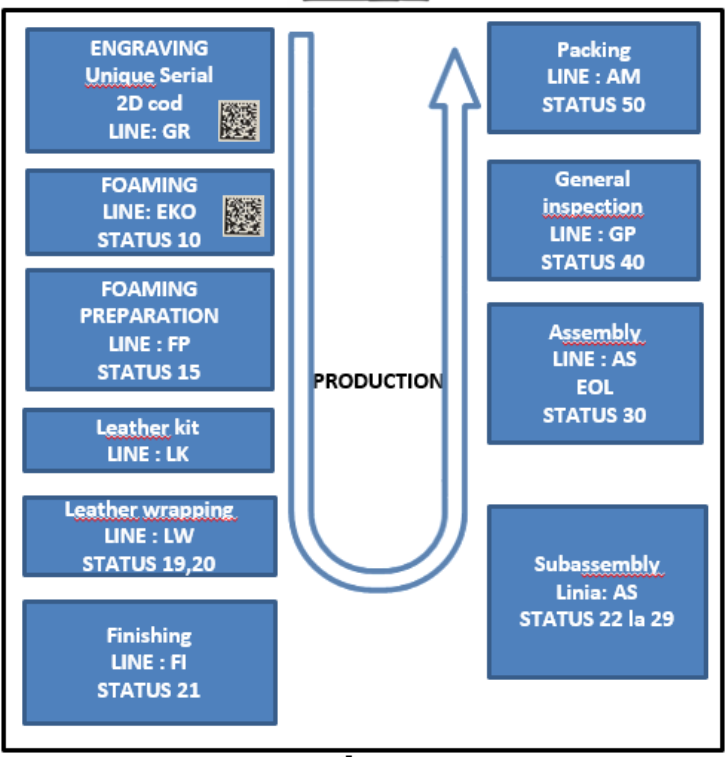

Fig.2: Status allocation

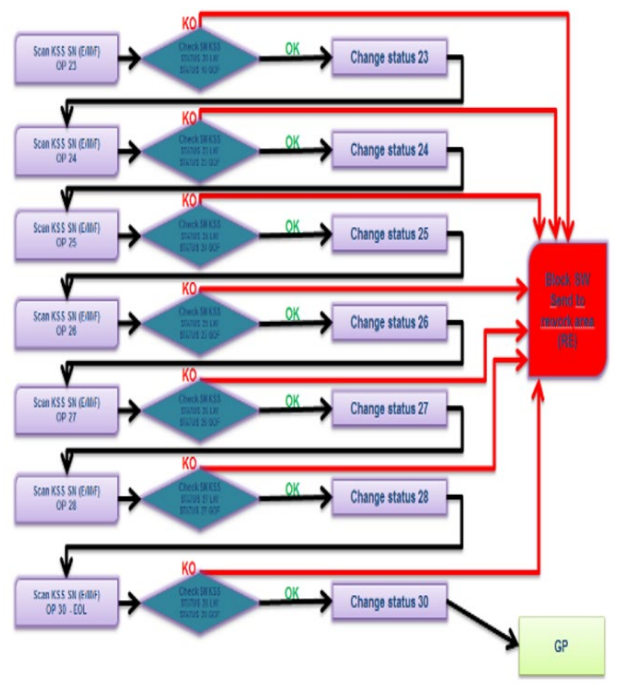

Fig. 3: Status allocation rework condition
- Producer checks the feasibility of production;

- Producer checks the allocation of materials;

- Producer checks the availability of sequencing racks;

- If feasibility is confirmed, producer launches the production and updates the TTM databases:
- Serial number
- Characteristics values.

Traceability confirms automatically in producer's ERP (Enterprise Resource Planning) [16] the availability of the product and in customer portals the characteristics results.

The complete workflow is analyzed in figure 3 , status allocation rework condition.

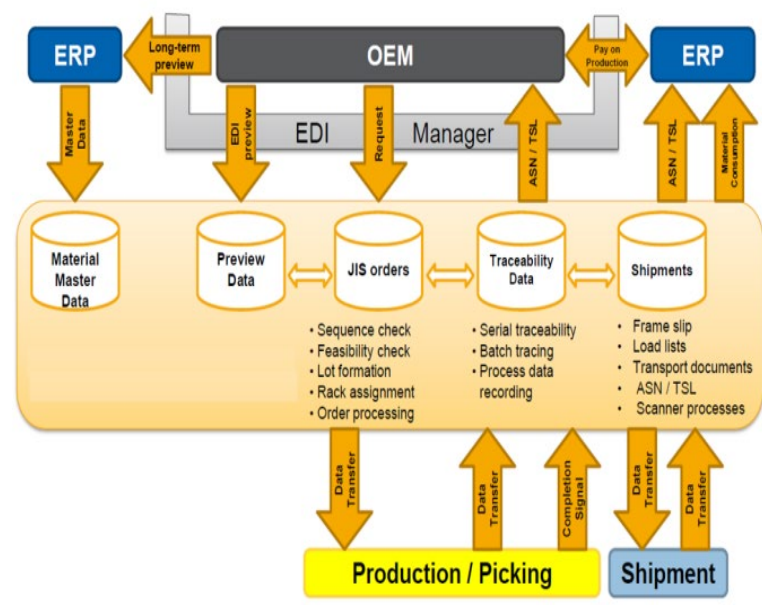

Fig.4: The JIS/JIT Traceability Module

The functions of the software are consisting in monitoring the parameters and assuring that all previous phases are executed correctly. The quality concept of "do not accept defects, do not produce defects, do not deliver defects" is embedded in the traceability software. Additionally the traceability software must be fully integrated with the ERP and the ERP must be connected to the OEMs supply chain software, as stated per figure 4, the JIS/JIT Traceability Module.

Serial Traceability, Batch Data, Process Data is connected to JIS orders and customer confirmations. In order to execute the full traceability processes a complete system configuration must be done from the design phase and include various features.

In order to assure the succession of products into all containers delivered to the final client, containers which are to be unpacked by industrial robots directly on the final assembly line, where the robot has the access in the right succession of the products according to the manufacturing program of the line.

This conditions the supplier to be connected permanently with the final client's system in order to be able to synchronize the production and packing of 
steering wheels with the orders of the final user and with production program of the plant.

This solution is an extended module of TTM (Total Traceability Management) installed at the supplier. This traceability system is adapted to the JIS/JIT procedures concept specific to steering wheel production assisted by the robots.

It fulfillments the traceability condition between the shop floor PLC and the PC (Fig.5). The safety and the key parameters are stored in pre-assembly stations PC1, PC2, PC3 based on the customer and legal agreements (examples: Airbag Communication, Horn Functions, and Geometrical Parameters, etc.). The sequencing is realized in station 20 (for example) where the pre-assembled components are built in to match the customer sequence (Fig. 6).

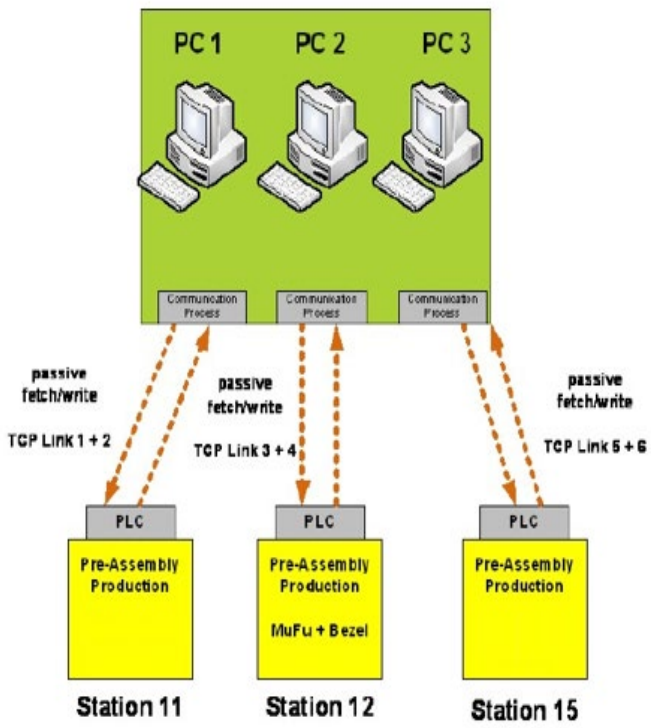

Fig. 5: PLC Communication with TTM

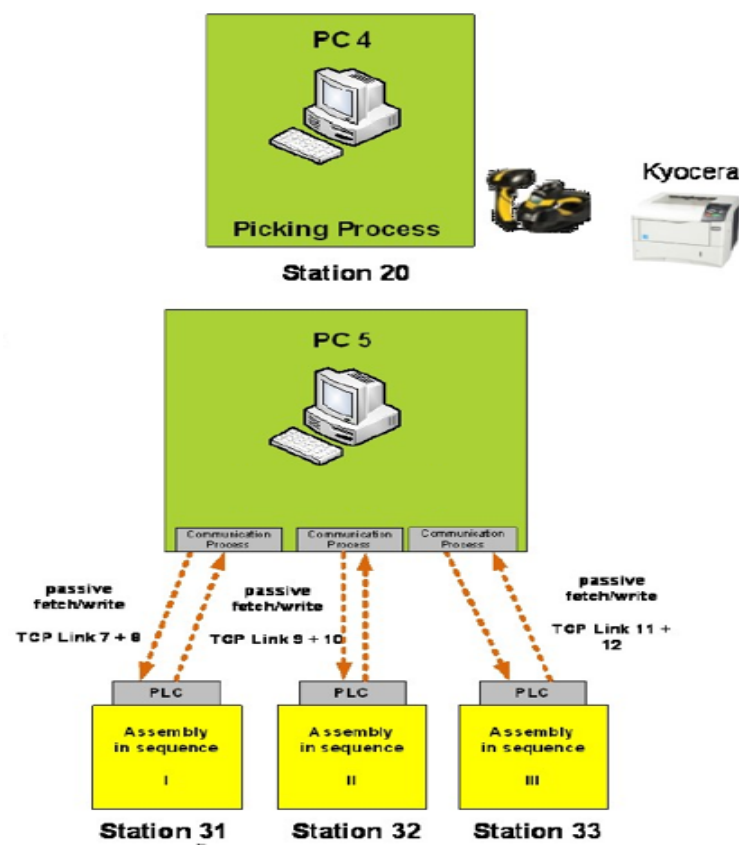

Fig.6: TTM produces JIS
Traceability associates the sub-assembly by scanning avoiding the allocation of a wrong component. This operation is coordinated by traceability software which is guiding the operator.

The customer specific labels for shipping are printed. Packing List is the customer sequence sent for a daily delivery buy the customer. The containers are travelling between the OEM and the producer. Once the order is completed the container will receive a new sequence need which translated in Traceability will generate a new pick list for the production process, as per figure 7 the JIS allocation.

During the allocation operations the traceability software is checking that all the process parameters are correctly confirmed and saved in the database.

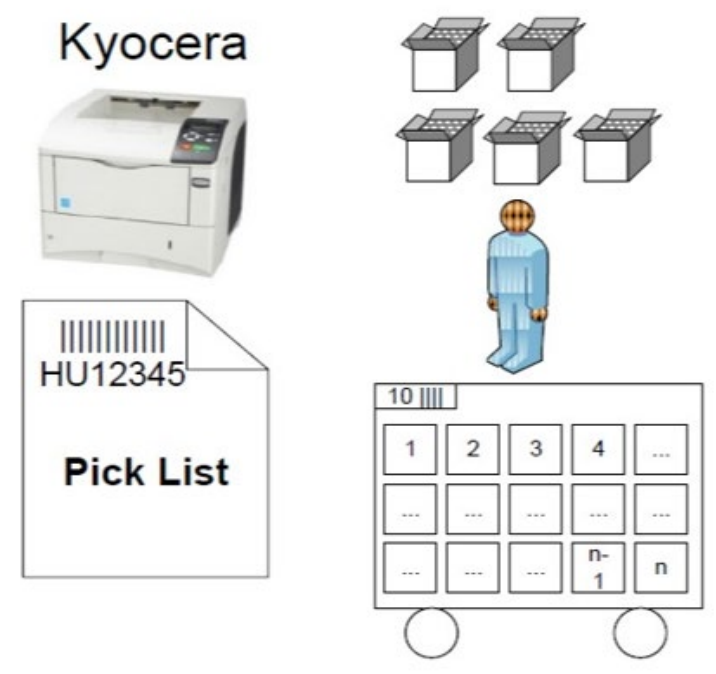

Fig.7: JIS Allocation

\section{Conclusion}

The traceability at maxim level (TTM-Total Traceability Management) is able to solve the following major targets: impeccable quality of the products, total flexibility as response to the customer requests, delivery terms into the contract limits, delivery of the products in JIT / JIS conditions.

The main problem of the current steering wheel processes is that are built for batch production.

The die-casting, foaming, leather wrapping, assembly and packing where designed for batch production. This production system enters in conflict with the increased product complexity and diversity. The solution for high diversity and complexity is the TTM (Total Traceability Management). It is a software solution which through its algorithms is solving the main challenges:

- Assuring the right Quality Parameters Error Proofing;

- Assuring the right deliveries through Communication with customer and local ERPs;

- Assuring the process control through software communication with equipment and operators;

- Assuring the fast setup through immediate access to inventory and information; 
- Is preparing the processes for IoT production.

This production system enters in conflict with the increased product complexity and diversity. The solution for high diversity and complexity is the TTM.

Additionally at the end of 2018, "S196 - 8.5.2.1 Identification and traceability - supplementary - IATF" was launched. This supplement contains clear obligations for suppliers in the automotive industry $[17,18]$. These are listed in the supplement as follows:

- Identification of the non-compliant and / or suspicious product;

- Segregation of non-compliant and / or suspicious product;

- Ability to comply with legal requirements regarding response times in case of withdrawals from the market or blocking of stocks in the market;

- The control of records must be organized in such a way as to comply with the legal requirements regarding response times in the event of withdrawals from the market or blocking of stocks in the market;

- Identification of products that must be with a unique serial code, if specified by the customer or if it is a legal requirement;

- Traceability requirements are extended to products delivered with critical, unsafe, or legal characteristics.

The achievement of the above main objectives, IATF conformity, characteristics traceability and JIS/JIT production is possible only through traceability software.

The authors of this article worked at the concept of JIS creation, realization and implementation on two over mentioned factories in Romania. Their work was more complex, because we are talking about of adaptation and modification of the technology, the information flow, the entire manufacturing philosophy according to the actually automation level of robotized production in automotive domain.

\section{References}

[1] Cleland-Huang, J., Gotel, O.C., Hayes, J.H., and others, (2014), - Software traceability: Trends and future directions, Conference: Proceedings of the on Future of Software Engineering, FOSE 2014, Hyderabad, India P.

[2] Peraldi-Frati, M.A., Albinet, A., (2010), Requirement traceability in safety critical systems, Conference: 1st Workshop on Critical Automotive Applications, Valencia, Spain.

[3] Qi, X. et al. (2014), OPC-MFuzzer: A novel multilayers vulnerability detection tool for OPC protocol based on fuzzy technology, International Journal of Computer and Communication Engineering, vol. 4, no. 4, pp 300-305.

[4] Hugh, J. (2008), Automatic Manufacturing Systems with PLC. [On line]. Available: http://www.freeinfosociety.com/media/pdf/2908 .pdf

[5] Tubbs, S. (2016), Programmable Logic Controller (PLC) Tutorial, Siemens Simatic S7-1200, Paperback. [Online]. Available: https://www.waterstones.com/book/programma ble-logic-controller-plc-tutorial-siemens simatics7-1200/stephen-philip-tubbs/9780981975368

[6] Lamb, F. (2019), Advanced PLC Hardware \& Programming: Hardware and Software Basics, Advanced Techniques \& Allen-Bradley and Siemens Platforms, Automation Consulting LLC, USA, pp 251-269.

[7] Serpanos, D. and Wolf M.C. (2018), Internet-ofThings (IoT), Systems Architectures, Algorithms, Methodologies, Springer, pp81-90.

[8] Stamatis, D.H. (2015), Quality Assurance: Applying Methodologies for Launching New Products, Services, and Customer Satisfaction (Practical Quality of the Future), $1^{\text {st }}$ edition, CRC Press, USA.

[9] Weschke, T. (2015), Design of the material flow and information flow processes in the operational area of an automotive supplier, (Gestaltung der Materialfluss - und Informationsflussprozess einbetrieblichen Bereich eines Automobilzulieferer), (German Edition), Munchen, GRIN Verlag. [4] Serpanos, D. and Wolf M.C. (2018), Internet-of-Things (IoT), Systems Architectures, Algorithms, Methodologies, Springer, pp81-90.

[10] BMW Platforms. (2016), Customer Specific Requirements Traceability BMW, [Online]. Available: https:/www.iatfglobaloversight.org/ oem-requirements/customer-specificrequirements/

[11] Porsche Platforms. (2018), Customer Specific Requirements Traceability Porsche, [Online]. Available: www.porsche-engineering.com

[12] Gerard, B. et al. (2002), Oracle E-Business, Suite Manufacturing \& Supply Chain Management, McGraw-Hill Education.

[13] Totally Integrated Automation Portal. [Online]. Available: www.siemens.com.

[14] German Association of the Automotive Industry (Verbandder Automobilindustrie). [Online]. Available: https://www.vda.de

[15] Tekin, E. (2014), A Method for Traceability and "As-built Product Structure" in Aerospace Industry, Procedia CIRP, vol. 17, pp. 351-355.

[16] Enterprise application software for ERP and internet of things. [Online]. Available: https://www.sap.com/products/iot-dataservices.html

[17] Ambrose, P. (2016), IATF 16949:2016 Plus ISO 9001; and (2015): Assessment (Audit), Guide and Checklist, 2nd edition, Amazon.

[18] IATF 16949: (2016) - Lead Auditor Training for Automotive Quality Management Systems. [Online]. Available: www.iatf.org 